\title{
Eye tracking y usabilidad en ambientes informacionales digitales: revisión teórica y propuesta de procedimiento de evaluación
}

\author{
Eye tracking and usability in digital informational \\ environments: theoretical review and \\ evaluation procedure proposal
}

\author{
Sandra Milena ROA-MARTÍNEZ1 (iD) 0000-0002-2271-6101 \\ Silvana Aparecida Borsetti Gregorio VIDOTT|2 (D) 0000-0002-4216-0374
}

\begin{abstract}
Resumen
Considerando la tecnología eye tracking como método de colecta de datos originados por el registro del seguimiento visual de las personas, se propone como objetivo de este trabajo orientar conceptual y metodológicamente el desarrollo de futuras investigaciones que pretendan usar esta tecnología para la evaluación de la usabilidad en ambientes digitales informacionales. Fue usado un enfoque metodológico descriptivo y prospectivo para el desarrollo de este trabajo, a partir de un levantamiento bibliográfico, análisis de literatura y experiencias en trabajos previos. Como resultado, se obtuvo un conjunto de pasos que constituyen una propuesta de procedimiento para evaluación de aspectos de usabilidad usando la tecnología eye tracking. Se destaca que se encuentra un amplio panorama y configuraciones para evaluar usabilidad usando eye tracking. El procedimiento propuesto pretende guiar futuras investigaciones relacionadas; además se presenta un consolidado de métricas con un significado en términos de evaluación de aspectos de usabilidad. Concluyendo, entre los principales aspectos de usabilidad medidos durante la evaluación por métricas de eye tracking están: eficiencia en la búsqueda, interfaz y visibilidad y que el procedimiento propuesto, además de guía, garantizará que los objetivos propuestos en este tipo de investigaciones sean claramente formulados y cumplidos al igual que la validez de sus resultados.
\end{abstract}

Palabras clave: Procedimiento de evaluación. Seguimiento ocular. Usabilidad.

\begin{abstract}
Defining eye tracking technology as a method of data collection originated by the registration of people's visual tracking, this work's objective is to guide the conceptual and methodological development of future research that intends to use this technology for the evaluation of usability in informational digital environments. For this work's development, we used a descriptive and prospective methodological approach, based on a bibliographic survey, literature analysis, and previous work experiences. As a result, we obtained

1 Universidad del Cauca, Facultad de Ingeniería Electrónica y Telecomunicaciones, Departamento de Sistemas. Sector Tulcán, Oficina 108, Edifício IPET, Popayán, Cauca, Colombia. Correspondencia para/Correspondence to: S. M. ROA-MARTínEZ.E-mail:<smroa@unicauca.edu.co>.

2 Universidade Estadual Paulista Júlio de Mesquita Filho, Faculdade de Filosofia e Ciências, Departamento de Ciência da Informação. Marília, SP, Brasil. Apoyo: Universidad del Cauca y Conselho Nacional de Desenvolvimento Científico e Tecnológico (Proceso n. 311936/2016-4). Recibido el 20 de agosto del 2019, re-presentada el 14 de noviembre de 2019 y aprobado el 3 de enero del 2020.
\end{abstract}

Como citar este artículo/How to cite this article

Roa-Martínez, S. M.; Vidotti, S. A. B. G. Eye tracking y usabilidad en ambientes informacionales digitales: revisión teórica y propuesta de procedimiento de evaluación. Transinformação, v. 32, e190067, 2020. Doi: http://dx.doi.org/10.1590/1678-9865202032e190067 
a set of steps that constitute a proposal of procedure for the evaluation of usability aspects of the eye tracking technology. It is worth noticing that there is a broad panorama and configurations that can be used to evaluate usability within eye tracking. The procedure proposed aims to guide future researchers and projects in these issues, in addition to presenting a consolidated metrics with a meaning in terms of usability aspects' evaluation. To conclude, among the main usability aspects measured during the evaluation by eye tracking metrics are: efficiency in the search, interface and visibility, and that the proposed procedure, besides providing guidance, guarantees that the objectives proposed in this type of research are clearly formulated and fulfilled, as are the validity of their results.

Keywords: Evaluation procedure. Eye tracking. Usability.

\section{Introducción}

El avance de las tecnologías y su aplicación como instrumento de apoyo en diversas áreas de conocimiento favorece el desarrollo de investigaciones interdisciplinarias. Es interesante resaltar que desde la Ciencia de la Información ya han sido abordadas investigaciones para la medición de los aspectos de usabilidad de ambientes informacionales digitales desde diferentes perspectivas, las cuales involucran diversos métodos y tipos de (re) colección, tratamiento y análisis de los datos. Algunas cuestiones estimadas cuando se realizan estudios de usabilidad con la tecnología eye tracking en Ciencia de la Información no quedan claras; por ejemplo, si la evaluación debería ser inductiva o deductiva, o si el análisis de los datos debe ser cualitativo o cuantitativo.

Lo anterior lleva a investigaciones que no contemplan el amplio panorama y configuraciones que pueden darse a partir del tipo de enfoque metodológico, potencial de las métricas y análisis de datos usados para medir Experiencia de Usuario (UX, UserExperience), usabilidad, findability u otros elementos en los ambientes informacionales digitales que involucren al usuario. Considerando que la usabilidad está contenida por la experiencia de usuario, la evaluación de aspectos de usabilidad implica una contribución en la evaluación de UX (destacando que las medidas de usabilidad objetivas dadas por los datos obtenidos con la tecnología eye tracking, foco de este trabajo, no contemplan elementos subjetivos de evaluación de UX y de findability) que se aborda y continúa siendo área de estudio en otros trabajos.

En vista de todo esto, surge la necesidad de iniciativas y prácticas vinculadas con el uso de la tecnología de eye tracking para medir aspectos de usabilidad que puedan ser propiciadas desde la Ciencia de la Información.

\section{Procedimientos Metodológicos}

Con este trabajo se busca orientar el desarrollo de futuras investigaciones que pretendan usar la tecnología eye tracking para la evaluación de aspectos de usabilidad en ambientes informacionales digitales. Para ello, se utilizó un enfoque metodológico descriptivo a partir de un levantamiento y revisión bibliográfica de métricas que permiten medir aspectos de usabilidad utilizando la tecnología eye tracking y otros elementos de índole teórica y procedimental. Posteriormente, un enfoque prospectivo permitió proponer un conjunto de pasos para constituir un procedimiento de evaluación de usabilidad usando eye tracking que contempla elementos conceptuales y metodológicos.

Este artículo aborda inicialmente aspectos teóricos sobre temáticas tales como la evaluación de usabilidad y terminología asociada a eye tracking, así como el uso de la tecnología eye tracking en evaluaciones de aspectos de usabilidad. También se presenta un referencial bibliográfico actualizado, pertinente y relativo a las métricas para la evaluación de usabilidad con esta tecnología, el cual comprende una ventana temporal desde 1950 hasta 2013, construido principalmente a partir de los trabajos de Jacob y Karn (2003) y de Sharma y Dubey (2014), destacándose la primera aplicación de eye tracking conocida actualmente como ingeniería de la usabilidad que fue propuesta 
por Fitts, Jones y Milton (1950), y estudios como el de Just y Carpenter (1976), pionero en relacionar elementos de eye tracking tales como las fijaciones a algunos procesos cognitivos específicos. Estos primeros trabajos en el área de eye tracking se encuentran en idioma inglés y fueron abordados principalmente por profesores del área de psicología en Estados Unidos.

\section{Marco Teórico}

La Experiencia de Usuario, según la Norma ISO 9241-210 (Internacional Standardization Organization, 2010) contempla las percepciones y respuestas personales resultantes del uso de un producto, sistema o servicio, incluyendo las emociones de los usuarios, creencias, preferencias, comportamientos y respuestas físicas y psicológicas entre otros elementos, que suceden antes, durante y después del uso.

En la misma Norma ISO 9241-210 (International Standardization Organization, 2010) la Usabilidad es la "[...] medida en que un sistema, producto o servicio puede ser usado por usuarios concretos para alcanzar objetivos específicos con eficacia, eficiencia y satisfacción en un contexto de uso dado".

A partir de estas definiciones de la Norma ISO, que destacan adicionalmente que los criterios de usabilidad pueden ser usados para evaluar algunos aspectos de la experiencia de usuario, y vista la usabilidad y UX entrelazadas, se destaca que: "[...] la evaluación UX implica el aumento de los métodos existentes para la evaluación de usabilidad. [...] Aunque el componente subjetivo de la usabilidad (es decir, la satisfacción) puede verse como parte de la evaluación de UX, UX aborda una gama de otras cualidades subjetivas" (Vermeeren et al., 2010, p. 522).

Además, la usabilidad también se define como la percepción de la consistencia, organización, eficiencia, facilidad de uso y cómo de intuitivo es el proceso de completar una tarea en particular dentro de un sistema (Baeza-Yates; Rivera Loaiza; Velasco Martín, 2004).

Para Roa-Martínez (2019), pueden establecerse varias relaciones entre la usabilidad y findability y, aunque cada concepto tiene objetivos diferentes, comparten el hecho de estar centradas en el usuario y sus necesidades informacionales al igual que en las interacciones con los ambientes informacionales digitales.

En cuanto que findability "[...] está relacionada con la calidad de respuesta que los usuarios consiguen, a partir del momento que definen una búsqueda para encontrar lo que están requiriendo" (Roa-Martínez, 2019, p. 84) y la interfaz es considerada como punto de entrada de los procesos de búsqueda y recuperación de la información e interacción del usuario, la cual puede ser evaluada por la usabilidad, entonces al medirlas y ser complementarias la usabilidad propiciaría la findability y viceversa, debido a que, por ejemplo, un ambiente para ser usable deberá ser encontrado previamente.

Específicamente, la evaluación de la usabilidad para Dias (2003, p. 42): "[...] debe verificar el desempeño (eficacia y eficiencia) de la interacción hombre-computador y obtener indicios del nivel de satisfacción del usuario, identificando problemas de usabilidad durante la realización de tareas específicas en su contexto de uso". Dicha evaluación puede realizarse en diferentes momentos, es decir, durante el inicio, la validación o en un período intermedio de la construcción de un ambiente informacional digital.

En este sentido, los ambientes informacionales digitales serán los productos y como medidas de usabilidad en este trabajo se considerarán la eficacia y eficiencia. De esta forma, la eficacia se centra en la cantidad de usuarios que realizan una tarea de forma exitosa sin ayuda del evaluador, mientras que la eficiencia considera los tiempos requeridos por los usuarios para completar una tarea, contabilizando solo aquellos que finalizan con éxito. 
Para Nielsen y Loranger (2007), las principales dificultades de usabilidad que se deben evaluar por desencadenar confusión y descontento entre los usuarios se clasifican por su gravedad (de mayor a menor) en: búsqueda, capacidad de localización, diseño de página o interfaz, información, soporte de tareas y diseño sofisticado, entre otras.

Entre algunos métodos usados para medir usabilidad, según Ivory y Hearst (2001), se encuentran los de inspección (recorrido cognitivo, evaluación heurística, etc.), de pruebas (pensar en voz alta, registro visual, etc.), de consulta (grupo focal, observación de campo, etc.) y por modelos y simulaciones.

Dentro de las técnicas existentes para estimar usabilidad se encuentra eye tracking, una tecnología que registra los movimientos oculares de un individuo, principalmente con la finalidad de determinar anormalidades o la interacción frente a estímulos o tareas definidas previamente. Esta finalidad se puede medir mediante el análisis de los datos generados durante el uso o aplicación de dicha tecnología (Duchowski, 2002).

Para realizar este registro, eye tracking cuenta con un componente conocido como eye tracker, un "[...] dispositivo que permite realizar un seguimiento y registro visual de un individuo a través de la transmisión de rayos infrarrojos y una cámara. La posición del ojo es identificada mediante cálculos realizados por el software que viene con los sistemas de seguimiento ocular" (Vidotti et al., 2016, p. 3741).

El comportamiento visual estará influenciado por: (1) cualquier elemento que capte la atención para ser observada (atención de abajo hacia arriba), orientada por el estímulo y (2) la intención voluntaria de observar algo, orientada por el conocimiento y basada en experiencias y expectativas (atención de arriba hacia abajo), destacando que: "[...] la misma persona mirará el mismo objeto de manera diferente si se le da una tarea diferente [...] Es la atención de arriba hacia abajo la responsable de estas diferencias" (Bojko, 2013, p.14).

Por ello, de manera significativa, los movimientos o el comportamiento de los ojos estarán influenciados por un estímulo o tarea, que está asociado a un nivel alto o bajo del proceso de cognición y estos movimientos podrán ser voluntarios (dependientes de experiencias o expectativas del individuo). Asimismo, tiene lugar otra dependencia del comportamiento visual, asociada al tipo de tarea que debe ser realizada.

En cuanto a los métodos para el análisis de datos generados por eye tracking, se destacan los estadísticos y visuales. El análisis estadístico proporciona principalmente resultados cuantitativos, mientras que las técnicas de análisis visual se orientan más hacia un modo cualitativo de estudiar los datos. La tecnología de eye tracking se entiende como un método de naturaleza cuantitativa usado para recolección de datos que son analizados y vinculados con tareas asociadas a actividades cognitivas (Cooke, 2004).

En este contexto, las métricas son técnicas usadas como unidades de medida para el análisis de datos generados por el software de eye tracking. Para entenderlas, se especifican en el Cuadro 1 los términos a partir de los cuales se definen dichas métricas.

Algunos de estos términos se representan en la Figura 1, donde las rutas o recorridos de exploración (scanpath) de cada usuario están definidos por un color (indicada en fucsia para un usuario, secuencialmente siguiendo la numeración que comienza en 1). El tamaño de algunos círculos es mayor, lo cual indica que el tiempo de la fijación del usuario en esa área fue mayor (nótese la diferencia de tamaño, por ejemplo, entre el círculo 49 y el círculo 30). Adicionalmente, se observa en la Figura 1, un ejemplo de la definición de un estímulo dentro del área de interés relacionado con los trabajos presentados en eventos.

El interés en el uso de métricas o visualizaciones para el análisis de datos generados por la tecnología eye tracking es proporcional al interés por el uso de esta tecnología, el cual, entre otros elementos, es creciente dados los avances tecnológicos y la accesibilidad a este instrumento (Lupu; Ungureanu, 2013).

Entre los tipos de métricas asociadas a estos términos se encuentran: (a) Métricas derivadas de fijaciones: generalmente utilizadas en estudios cuantitativos con eye tracking, ofrecen una idea general sobre el desarrollo de 
Cuadro 1. Descripción de términos asociados a las métricas.

\begin{tabular}{|c|c|c|}
\hline Término & Descripción & Visualización (representación gráfica) \\
\hline Fijación (Fixation) & $\begin{array}{l}\text { Es el periodo de tiempo durante el cual los ojos están relativamente } \\
\text { "quietos", es decir, el tiempo en el que un usuario procesa (codifica) } \\
\text { la información que está observando (Sharma; Dubey, 2014). Para ser } \\
\text { considerada una fijación, dependerá del tiempo mínimo necesario } \\
\text { establecido y de la amplitud del movimiento máximo aceptable } \\
\text { (Giannotto, 2009). Estos parámetros están determinados por el algoritmo } \\
\text { utilizado en el software de eye tracking utilizado. }\end{array}$ & \\
\hline Sacada (Saccade) & $\begin{array}{l}\text { Es el movimiento usado para reposicionar la fóvea del ojo de un punto de } \\
\text { interés a otro (Giannotto, 2009). Puede ser medida como el intervalo de tiempo } \\
\text { entre dos fijaciones o como la distancia de una fijación a otra, dependiendo del } \\
\text { software utilizado para su cálculo. }\end{array}$ & \\
\hline $\begin{array}{l}\text { Ruta de exploración } \\
\text { (Scanpath) }\end{array}$ & $\begin{array}{l}\text { Es considerada una medida derivada de las fijaciones y sacadas que } \\
\text { representa la ruta o secuencia ordenada de fijaciones y sacadas realizadas } \\
\text { por los ojos de un usuario. }\end{array}$ & \\
\hline Área de interés & $\begin{array}{l}\text { Espacio o región visual de interés, donde será establecido el estímulo } \\
\text { objeto de estudio. Estas áreas son determinadas por el investigador. }\end{array}$ & \\
\hline Mirada (Gaze) & $\begin{array}{l}\text { Serie de fijaciones consecutivas y sacadas dentro de un área de interés. } \\
\text { Puede medirse la duración de una gaze a partir de la suma de cada una } \\
\text { de las duraciones de las fijaciones que la constituyen y la posición de la } \\
\text { gaze con el promedio de la posición de las fijaciones en el área de interés } \\
\text { (Giannotto, 2009). }\end{array}$ & Ver Figura 1 \\
\hline
\end{tabular}

Fuente: Elaborado por los autores (2019).

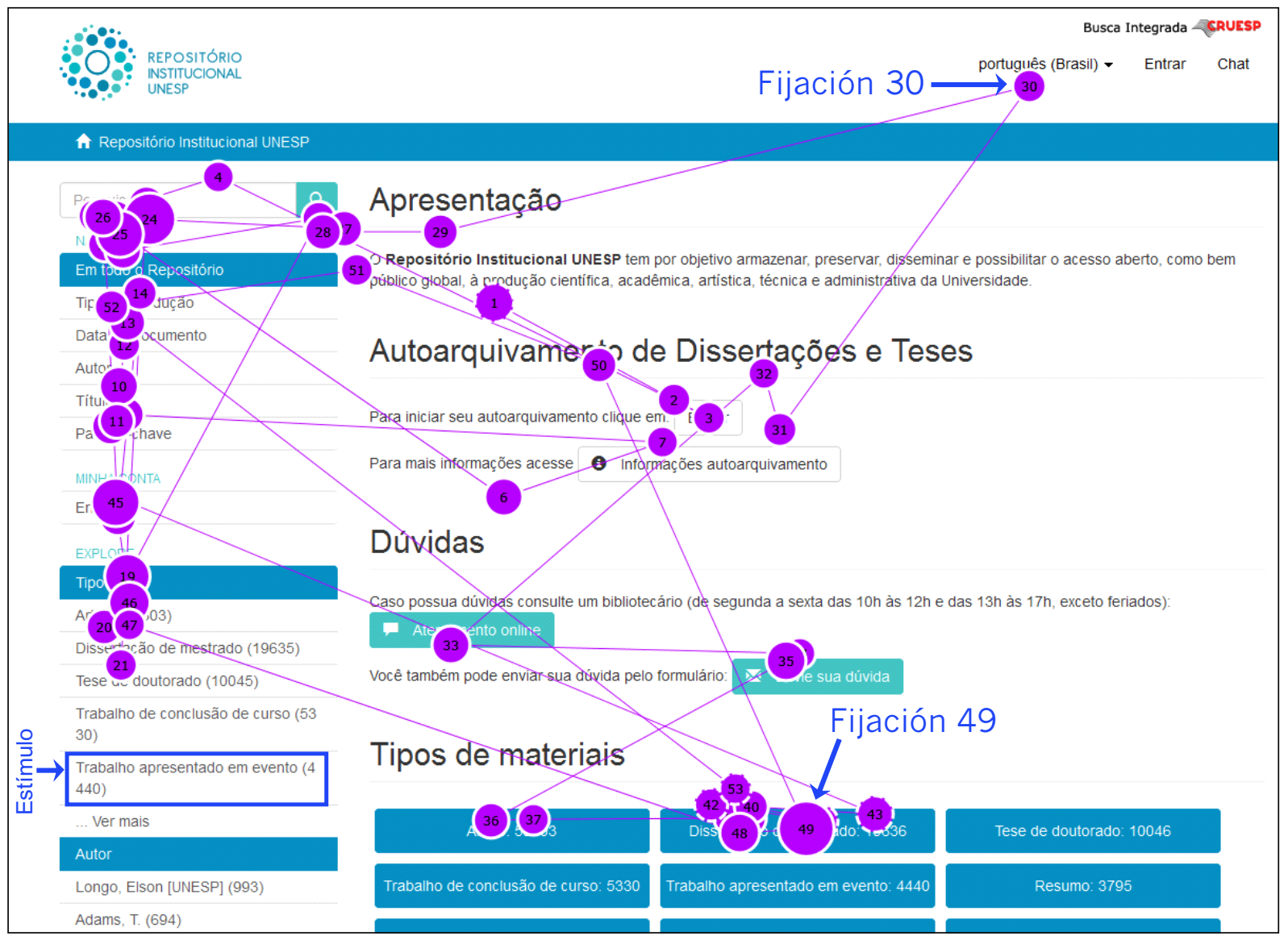

Figura 1. Diagrama de la mirada (Gaze plot) de un usuario dentro de un ambiente informacional digital.

Fuente: Elaborado por los autores desde la página de início del Repositório Institucional Unesp (Universidade Estadual Paulista, 2019). 
una tarea. En esta categoría se encuentra un alto número de métricas identificadas por autores como Goldberg et al. (2002), Jacob y Karn (2003) y Poole y Ball (2005) quienes afirman que miden principalmente la eficiencia en la búsqueda de informaciones o la dificultad en la utilización de elementos de la interfaz; (b) Métricas derivadas de sacadas: son menos comunes en evaluaciones de usabilidad (Giannotto, 2009). Pueden indicar la calidad de los elementos visuales en el estímulo o la amplia búsqueda visual; por tanto, estas métricas pueden indicar dificultades como la codificación visual (Kurzhals et al., 2015); (c) Métricas derivadas del scanpath: pueden adquirir información sobre estrategias visuales de lectura o identificar problemas específicos con el diseño de la visualización durante la tarea (Kurzhals et al., 2015).

Para autores como Bojko (2013), eye tracking puede proveer información para mejorar las recomendaciones de diseño mediante la detección y explicación de cuestiones de usabilidad. Además, proporciona formas para medir la experiencia de usuario y comparar diseños por medio de medidas de atracción y ejecución.

Entre las técnicas de visualización más referenciadas en la literatura (Kurzhals et al., 2015) se hallan los mapas de atención y diagramas de mirada (gaze plots). Los mapas de atención despliegan la distribución espacial de los datos de eye tracking sobre un estímulo estático que se encuentra en una determinada área de interés (pueden ser datos de un individuo o de varios participantes), perdiendo esta técnica el componente temporal de los datos. Los gaze plots, por el contrario, proveen una perspectiva espaciotemporal que puede ser usada, por ejemplo, para identificar estrategias potenciales de lectura. Sin embargo, puede ser difícil la interpretación de los datos con estas técnicas, sea por el incremento en la longitud de la ruta de exploración (scanpath) o por la aplicación en múltiples participantes.

Los aspectos de usabilidad usando la tecnología eye tracking pueden ser evaluados de manera cuantitativa, cualitativa o mediante una combinación de ambas. Vale destacar que las medidas cuantitativas serán analizadas por métodos estadísticos y que las visualizaciones y métricas pueden ser usadas a partir de los datos generados por esta tecnología.

\section{Métricas de Eye Tracking para evaluar usabilidad}

Durante el levantamiento y revisión bibliográficos realizados, se revisaron los trabajos relacionados al uso de eye tracking en evaluaciones de usabilidad referenciados en las investigaciones de Jacob y Karn (2003) que comprenden el período entre 1950 y 2000, con un total de veinticuatro (24) trabajos. Adicionalmente, se analizaron catorce (14) trabajos contemplados por Sharma y Dubey (2014) desde 1998 hasta 2013.

La frecuencia de uso sobre las principales medidas utilizadas en esta revisión se basó en: Miradas o Gaze (20), Fijaciones (15), Rutas de exploración o Scanpath (12), Sacadas (6) y Pupila (2). Las medidas asociadas a gaze (serie de fijaciones consecutivas dentro de un área de interés) que presentaron una mayor frecuencia están asociadas por su definición a las fijaciones que también presentan una alta frecuencia.

A continuación, en el Cuadro 2, se describen las principales métricas basadas en estas medidas para los datos cuantitativos que proporciona la tecnología eye tracking. Para cada una de las veintisiete (27) métricas identificadas se indica la referencia al autor seminal de dicha métrica. Adicionalmente, se asocia uno o más aspectos de usabilidad que puede o pueden evaluarse con cada métrica y una breve descripción relativa a la evaluación del aspecto de usabilidad que cada métrica pretende.

Las medidas asociadas a la pupila generalmente están determinadas por factores tales como la luminosidad del ambiente y la contaminación; por lo tanto, son menos usadas en trabajos de eye tracking (Goldberg; Wichansky, 2002). 
Entre los aspectos más evaluados por las métricas del Cuadro 2 se destacan los siguientes aspectos de un ambiente informacional digital: la eficiencia en la búsqueda, la interfaz, la atracción, la visibilidad o la importancia de elementos y de la información.

Asimismo, fue considerada la revisión de literatura realizada por Lund (2016) con respecto a la aplicación de eye tracking en la Ciencia de la Información. Los trabajos se clasifican en cuatro paradigmas o tópicos de investigación: Lectura (Reading), Percepción natural de la escena (Natural scene perception), Búsqueda visual (Visual search) y usabilidad (Usability). Además, se percibe un aumento del uso de esta tecnología en los últimos años, donde la mayoría de los estudios con eye tracking son de usabilidad (66\%) y utilizan las métricas mencionadas para su evaluación.

Cuadro 2. Métricas utilizadas para estimar aspectos de usabilidad.

\begin{tabular}{|c|c|c|c|c|}
\hline Medidas & Métrica & Referencia & Aspecto de usabilidad & Descripción \\
\hline \multirow{4}{*}{$\begin{array}{l}\text { Mirada } \\
\text { (Gaze) }\end{array}$} & $\begin{array}{l}\text { Duración del gaze } \\
\text { sobre un área de } \\
\text { interés. }\end{array}$ & $\begin{array}{l}\text { Fitts, Jones, Milton } \\
\text { (1950). }\end{array}$ & Extracción de información. & $\begin{array}{l}\text { Una mayor duración indica dificultad en la } \\
\text { extracción o interpretación de la información } \\
\text { sobre elementos de la interfaz. }\end{array}$ \\
\hline & $\begin{array}{l}\text { Gaze (permanencia } \\
\text { o dwell). }\end{array}$ & $\begin{array}{l}\text { Mello-Thoms, Nodine, } \\
\text { Kundel (2002). }\end{array}$ & Atención en los objetivos. & $\begin{array}{l}\text { Una gaze larga sobre un área de interés significa } \\
\text { la anticipación a la ocurrencia de un evento o } \\
\text { atención en los objetivos. }\end{array}$ \\
\hline & Orientación del gaze. & Renshaw et al. (2003). & Diseño de interfaz. & $\begin{array}{l}\text { Retroalimenta sobre el éxito de las características } \\
\text { de diseño. }\end{array}$ \\
\hline & $\begin{array}{l}\text { Número de gazes } \\
\text { por área de interés. }\end{array}$ & Jacob y Karn (2003). & $\begin{array}{l}\text { Atracción o importancia } \\
\text { de un elemento. }\end{array}$ & $\begin{array}{l}\text { Un mayor número indica la importancia de los } \\
\text { elementos del área e interés. }\end{array}$ \\
\hline Pupila & Tamaño de la pupila. & Marshall (2000). & Carga de trabajo cognitiva. & $\begin{array}{l}\text { Un tamaño grande de las pupilas puede indicar } \\
\text { un mayor esfuerzo cognitivo. }\end{array}$ \\
\hline \multirow{5}{*}{ Sacadas } & Número de sacadas. & $\begin{array}{l}\text { Goldberg y Kotval } \\
\text { (1999). }\end{array}$ & Búsqueda. & $\begin{array}{l}\text { Un alto número de sacadas indica mayor } \\
\text { búsqueda de los elementos. }\end{array}$ \\
\hline & Amplitud de la sacada. & Goldberg et al. (2002). & Atracción de la información. & $\begin{array}{l}\text { Sacadas largas indican elementos más } \\
\text { significativos que captan la atención del usuario } \\
\text { marcada por la distancia de la sacada. }\end{array}$ \\
\hline & $\begin{array}{l}\text { Duración de la } \\
\text { sacada. }\end{array}$ & Vuori et al. (2004). & $\begin{array}{l}\text { Calidad de elementos } \\
\text { (imágenes). }\end{array}$ & $\begin{array}{l}\text { Alta duración de sacadas significa baja calidad } \\
\text { de imágenes, como bajo contraste o elementos } \\
\text { borrosos. }\end{array}$ \\
\hline & Sacadas regresivas. & Sibert y Jacob (2000). & Atracción de la información. & $\begin{array}{l}\text { Las regresiones de sacadas indican la presencia } \\
\text { de elementos menos significativos. }\end{array}$ \\
\hline & $\begin{array}{l}\text { Sacadas que revelan } \\
\text { cambios direccionales } \\
\text { marcados. }\end{array}$ & $\begin{array}{l}\text { Cowen, Ball, Delin } \\
\text { (2002). }\end{array}$ & $\begin{array}{l}\text { Objetivos del usuario. } \\
\text { Interfaz. }\end{array}$ & $\begin{array}{l}\text { Cambios bruscos superiores a } 90 \text { grados indican } \\
\text { que los objetivos del usuario cambiaron o que } \\
\text { el diseño de la interfaz no coincide con las } \\
\text { expectativas del usuario. }\end{array}$ \\
\hline \multirow{3}{*}{ Fijaciones } & $\begin{array}{l}\text { Duración de la } \\
\text { fijación. }\end{array}$ & Just y Carpenter (1976). & $\begin{array}{l}\text { Extracción de información. } \\
\text { Atracción de los elementos. }\end{array}$ & $\begin{array}{l}\text { Una duración de la fijación más larga significa } \\
\text { dificultad para extraer la información o que un } \\
\text { elemento es atractivo para el usuario. }\end{array}$ \\
\hline & $\begin{array}{l}\text { Número total de } \\
\text { fijaciones. }\end{array}$ & $\begin{array}{l}\text { Goldberg y Kotval } \\
\text { (1999). }\end{array}$ & Eficiencia en la búsqueda. & $\begin{array}{l}\text { Un mayor número indica búsqueda ineficiente, } \\
\text { que puede deberse a un mal diseño de interfaz. }\end{array}$ \\
\hline & $\begin{array}{l}\text { Fijaciones por área } \\
\text { de interés. }\end{array}$ & Jacob y Karn (2003). & $\begin{array}{l}\text { Percepción o importancia } \\
\text { del área de interés. }\end{array}$ & $\begin{array}{l}\text { Un mayor número de fijaciones en una } \\
\text { determinada área de interés indicará que es más } \\
\text { perceptible o importante con respecto a otras. }\end{array}$ \\
\hline
\end{tabular}


Cuadro 2. Métricas utilizadas para estimar aspectos de usabilidad.

\begin{tabular}{|c|c|c|c|c|}
\hline Medidas & Métrica & Referencia & Aspecto de usabilidad & Descripción \\
\hline \multirow{6}{*}{ Fijaciones } & $\begin{array}{l}\text { Fijaciones por área } \\
\text { de interés ajustado } \\
\text { a la longitud del } \\
\text { texto. }\end{array}$ & $\begin{array}{l}\text { Poole, Ball, Phillips } \\
\text { (2005). }\end{array}$ & $\begin{array}{l}\text { Palabras por leer en el área } \\
\text { de interés. } \\
\text { Reconocimiento de los } \\
\text { elementos en el área de } \\
\text { interés. }\end{array}$ & $\begin{array}{l}\text { La tasa calculada a partir de la división del } \\
\text { número de fijaciones en el área de interés entre } \\
\text { el número de palabras del texto puede indicar } \\
\text { (cuando el número de fijaciones es alto) que } \\
\text { son muchas palabras por leer (cuando el área de } \\
\text { interés enmarca solo texto) o que los elementos } \\
\text { son difíciles de reconocer. }\end{array}$ \\
\hline & $\begin{array}{l}\text { Densidad espacial } \\
\text { de la fijación. }\end{array}$ & $\begin{array}{l}\text { Cowen, Ball, Delin, } \\
\text { (2002). }\end{array}$ & $\begin{array}{l}\text { Búsqueda enfocada y } \\
\text { eficiente. }\end{array}$ & $\begin{array}{l}\text { La concentración de fijaciones en un área } \\
\text { pequeña indica una búsqueda enfocada y } \\
\text { eficiente. } \\
\text { La dispersión espacial de las fijaciones al contrario } \\
\text { significan búsqueda ineficiente o exploratoria. }\end{array}$ \\
\hline & $\begin{array}{l}\text { Fijaciones repetidas } \\
\text { sobre el objetivo en } \\
\text { el área de interés. }\end{array}$ & $\begin{array}{l}\text { Goldberg y Kotval, } \\
\text { (1999). }\end{array}$ & $\begin{array}{l}\text { Significado o visibilidad de } \\
\text { la información. }\end{array}$ & $\begin{array}{l}\text { Un número alto de fijaciones repetidas sobre } \\
\text { el área de interés objetivo ya vista previamente } \\
\text { indica que tal objetivo tiene poco significado o } \\
\text { baja visibilidad. }\end{array}$ \\
\hline & $\begin{array}{l}\text { Tiempo para la } \\
\text { primera fijación en } \\
\text { el objetivo. }\end{array}$ & Byrne et al. (1999). & $\begin{array}{l}\text { Visibilidad o atracción de } \\
\text { los elementos. }\end{array}$ & $\begin{array}{l}\text { Poco tiempo para fijarse en el objetivo significa } \\
\text { que el elemento es visible para el usuario. }\end{array}$ \\
\hline & $\begin{array}{l}\text { Porcentaje de } \\
\text { participantes que se } \\
\text { fijan en el área de } \\
\text { interés. }\end{array}$ & Albert (2002). & $\begin{array}{l}\text { Visibilidad o atracción de } \\
\text { los elementos. }\end{array}$ & $\begin{array}{l}\text { Un bajo porcentaje indica que es necesario } \\
\text { resaltar o mover la información porque es poco } \\
\text { visible o con baja atracción. }\end{array}$ \\
\hline & $\begin{array}{l}\text { Fijaciones sobre el } \\
\text { objetivo dividido por } \\
\text { el número total de } \\
\text { fijaciones. }\end{array}$ & $\begin{array}{l}\text { Goldberg y Kotval } \\
\text { (1999). }\end{array}$ & Eficiencia en la búsqueda. & $\begin{array}{l}\text { Un bajo valor en esta tasa indica una poca } \\
\text { eficiencia en la búsqueda. }\end{array}$ \\
\hline
\end{tabular}

Fuente: Elaborado por los autores (2019).

\section{Procedimiento propuesto para evaluación de usabilidad usando Eye Tracking}

A partir del análisis detallado en las secciones anteriores se propone un procedimiento compuesto por un conjunto de pasos a seguir en futuros trabajos que involucran el uso de eye tracking para evaluaciones de aspectos de usabilidad de los ambientes informacionales digitales desde la Ciencia de la Información.

Paso 1

Inicialmente, debe definirse el "enfoque" con el cual se desarrollará la investigación. Para ello, según Cooke (2004), eye tracking es útil en investigaciones que estudien usabilidad, dado que agrega una dimensión de procesamiento cognitivo con respecto a otros proyectos realizados, pudiendo ser potenciada con la combinación de otros métodos (con enfoque en la ejecución, el comportamiento de usuario, entre otros). Este mismo autor, a partir de la propuesta de Goldberg et al. (2002), adoptada por Jacob y Karn (2003) con respecto a usabilidad, discute los tres tipos de enfoques que usualmente pueden utilizarse para el análisis del movimiento visual a través de eye tracking en usabilidad.

Estos enfoques son: (1) Top-down basado en una teoría o modelo cognitivo previo. Con este enfoque se han realizado principalmente trabajos con modelos afines a procesos de búsqueda y recuperación; (2) Bottom-up, en 
donde los investigadores no tienen una teoría o hipótesis previa correspondiente con una acción cognitiva, por cuanto las inferencias de comportamiento serán derivadas a partir de los datos obtenidos y que se utiliza cuando los investigadores están interesados en descubrir patrones que revelan los datos; (3) Top-down basado en una hipótesis que es previamente explicada y a partir de la cual se diseña el proyecto que se realizará para aprobarla o desaprobarla.

Este primer paso de la definición del enfoque será determinado por los datos. Si ya se tiene un conjunto de datos colectados y a partir de ellos se iniciará la investigación, entonces es un enfoque Bottom-up. Por el contrario, si previamente se plantea una hipótesis que será evaluada y para ello es necesaria la obtención de datos con la tecnología eye tracking para medir aspectos de usabilidad, corresponderá con un enfoque Top-down basado en hipótesis. Sin embargo, también puede ser de interés la confirmación de una teoría o modelo cognitivo y, para ello, se escoge también el enfoque Top-down con posterior obtención de los datos.

Paso 2

El siguiente paso será definir "el estímulo y la tarea" a presentar para quienes utilizarán el ambiente informacional digital, siendo necesario establecer las diferencias entre estos dos términos. Para Duchowski (2007), los movimientos oculares ocurren según los procesos cognitivos que pueden ser de bajo o alto nivel. Los de bajo nivel son aquellos donde la visión se posiciona de acuerdo con elementos que capturan su atención o "atractivos", es decir, orientados por el estímulo (stimulus-driven). Los de alto nivel son orientados por una tarea u objetivo (goal-driven), para lo cual se requiere un"recorrido"visual y conocimiento que permita que un objetivo sea alcanzado. "[..] las "cosas" registradas por los sentidos son llamadas estímulos [...] por ejemplo, un sitio web" (Bojko, 2013, p. 72).

Las tareas asociadas a intenciones de búsqueda que permitan determinar comportamientos de usuario en un ambiente informacional son, según Broder (2002), (1) Informacional: encontrar una información específica (por ejemplo, correo electrónico de contacto); (2) Navegacional: encontrar un sitio web determinado (por ejemplo, página de inicio de un banco donde se requiere de información para abrir una cuenta); y (3) Transaccional: realizar una determinada acción (por ejemplo, comprar entradas para ir al cine o descargar una imagen).

En una evaluación de usabilidad, la tarea será establecida como el objetivo que debe conseguir el usuario sobre un estímulo o conjunto de estímulos dados, que serán definidos usando la tecnología eye tracking y que seguirán la clasificación citada por Broder (2002). Un ejemplo de esto puede ser un área de interés en un menú (estímulo dentro de un ambiente informacional) y una tarea como la búsqueda de una determinada información en dicho ambiente, donde un objetivo es determinar si el usuario encuentra fácilmente dicha información cuando usa el ambiente en evaluación.

Paso 3

La técnica para evaluación de aspectos de usabilidad tratada en este trabajo ha sido eye tracking. Sin embargo, Bojko (2013) resalta que "combinar con otros métodos" (pensar en voz alta, cuestionarios, etc.) aporta beneficios tales como un mayor entendimiento, un conjunto más completo para chequeo de resultados y más información de un ambiente informacional digital con respecto a su usabilidad.

Paso 4

El próximo paso, a partir de la identificación del conjunto de métricas presentadas en el Cuadro 2, es "seleccionar" aquellas que se encuentran altamente vinculadas con los objetivos del proyecto. Estas métricas serán representadas en la tarea que realizarán los usuarios con la tecnología eye tracking y permitirán medir aspectos de usabilidad. 
Por otra parte, a partir de experiencias en trabajos realizados por los autores, se recomienda realizar una "evaluación cualitativa", como el "análisis de cada uno de los vídeos o grabaciones" generadas durante la realización de la tarea propuesta para cada uno de los participantes. Esto permitirá determinar cuándo se perdió el registro visual, en caso de que ocurra una pérdida, dado que desconocer este hecho altera los datos cuantitativos generados. Asimismo, es relevante revisar si efectivamente el usuario realizó la tarea propuesta, porque solo durante la revisión de algunas grabaciones puede encontrarse que algunos usuarios que responden afirmativamente con respecto a haber alcanzaron el objetivo propuesto, en realidad no lo consiguieron, lo cual, indiscutiblemente, también sesga las discusiones y conclusiones que se derivan a partir de los resultados.

Paso 6

En este paso, deberán "analizarse los datos cuantitativos" a partir del significado de las métricas seleccionadas y su correspondencia con los objetivos del proyecto. Estos datos son generados por el software de la tecnología eye tracking, y corresponderá al investigador dar significado a los datos colectados después de su análisis a la luz de las métricas. Por ejemplo, se puede tomar la métrica del número total de fijaciones y, según los valores arrojados por el software de eye tracking, determinar si dado un bajo número de estas durante la ejecución de los test realizados por los usuarios, se puede afirmar que la búsqueda en el ambiente informacional digital es eficiente y por ende representa un buen diseño de la interfaz. La descripción correspondiente a la evaluación de este aspecto de usabilidad del ejemplo está acorde con la métrica propuesta por Goldberg y Kotval (1999). Sin embargo, el análisis y significado a partir del dato cuantitativo deberán ser generados por el investigador.

\section{Paso 7}

Posteriormente, podrán "generarse visualizaciones de los datos". Las visualizaciones deben entenderse como el refuerzo de la "historia" que salió de las estadísticas (Bojko, 2013), es decir, como una forma de representación de resultados cuantitativos. Un ejemplo de utilización inadecuada de algunos tipos de visualizaciones es el uso de los mapas de calor, para indicar las zonas más visualizadas en las cuales se centró el registro visual del usuario. Este mapa puede ser interpretado incorrectamente por un investigador en aquellas zonas sin color, pudiendo concluir que no fueron visualizadas por el usuario (cuando el usuario en realidad sí visualizó esas zonas sin color, pero no el centro de atención).

En el ejemplo anterior, podría darse una evaluación parcialmente errónea cuando el objetivo es conocer el recorrido visual de un usuario. Por ello, sería recomendable utilizar otro tipo de visualización como, por ejemplo, un gaze plot. En consecuencia, es importante conocer claramente la interpretación que debe darse a cada tipo de visualización o método de análisis de datos que será usado.

En este contexto, Prisacari y Holme (2013) afirman que durante el uso de eye tracking para estimar usabilidad según los trabajos revisados en su investigación, es interesante notar que algunos métodos de análisis de datos son "poderosos", pero, en caso de ser incorrectamente aplicados e interpretados, pueden convertirse en "poderosos engaños".

A continuación, en la Figura 2, se resumen los pasos propuestos que han sido discutidos a lo largo de esta sección. Pueden ser considerados en futuros trabajos que pretendan estimar los aspectos de usabilidad en un ambiente informacional digital usando la tecnología de eye tracking y son el resultado del análisis y revisión discutidos en este trabajo.

Estos pasos, desde la definición del enfoque (dependerá de si ya se cuenta con los datos recolectados o no) para orientar la investigación hasta el cuidado que debe tener el investigador en la selección de las métricas para 


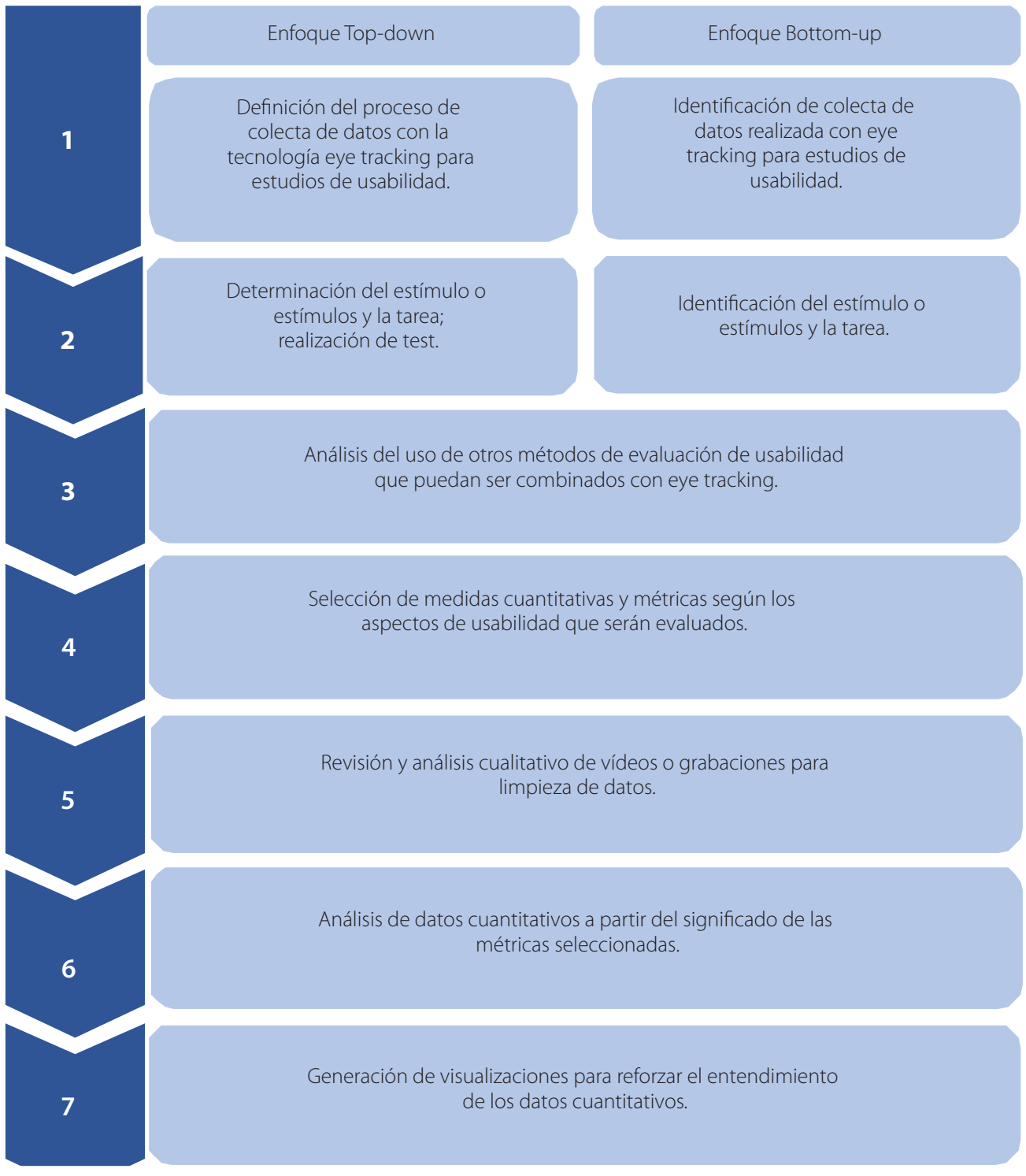

Figura 2. Pasos propuestos para la realización de trabajos que evalúan aspectos de usabilidad a través de la tecnología eye tracking. Fuente: Elaborado por los autores (2019).

medir el aspecto de usabilidad que desea estimar, son elementos que, principalmente a partir de la práctica y de antecedentes, pueden ser definidos con el propósito principal de que puedan abordarse nuevas investigaciones a partir de su uso.

\section{Conclusión}

Para determinar un método adecuado que permita desarrollar un proceso de investigación, deben analizarse las singularidades de cada proyecto. Es conveniente la caracterización del objeto de análisis y las diversas técnicas o mecanismos existentes, que deberán garantizar que los objetivos propuestos sean cumplidos y conlleven al avance y reproductibilidad de la ciencia en las investigaciones, como es el caso de la evaluación de usabilidad. 
El punto principal de este trabajo fue el análisis de la tecnología eye tracking como metodología de captura de datos de naturaleza cuantitativa e instrumento de evaluación de la usabilidad en ambientes informacionales digitales. A partir de una revisión bibliográfica sobre métricas, se logró identificar aquellas que permiten medir aspectos de usabilidad con esta tecnología.

Para el análisis estadístico y visualizaciones sobre datos recolectados con la tecnología eye tracking en ambientes informacionales digitales, se encontró que son usadas principalmente las métricas que evalúan aspectos tales como eficiencia en la búsqueda, interfaz, visibilidad, los cuales ocasionan confusión a los usuarios y forman parte de las cuestiones relevantes y conocidas de usabilidad por abordar.

Se construyó un procedimiento que contiene un conjunto de pasos recomendados para la ejecución de futuras investigaciones que pretendan el uso de la tecnología eye tracking para evaluar aspectos de usabilidad. El procedimiento propuesto considera aspectos metodológicos y conceptuales para la ejecución de futuros trabajos relacionados.

Vale destacar que, durante el análisis, diseño y creación de ambientes informacionales digitales deben tenerse en cuenta además de la usabilidad, la accesibilidad y findability (capacidad de encontrar), dado que el entorno no es solo el contenido y la estructura sino también la experiencia de usuario y por esto la importancia de estudios e investigaciones que involucren estas temáticas y tecnologías como eye tracking que apoyan procesos de recolección y evaluación con enfoque en el usuario. Por esto, futuros trabajos relacionados con el uso de eye tracking para la evaluación de findability, y por ende UX, podrían adaptar este procedimiento propuesto y configurar un conjunto de métricas específicas para los aspectos que se deseen evaluar en el paso 4 y para su posterior análisis e interpretación en el paso 6, conservando los demás pasos su finalidad orientada a findability o UX, si fuera el caso.

Finalmente, se concluye que es necesaria la combinación con otros métodos adicionales a la tecnología de eye tracking que evalúen la usabilidad, para garantizar una mayor verificación de resultados y más información de un ambiente informacional digital con respecto a la estimación de sus aspectos de usabilidad.

\section{Agradecimientos}

Al Grupo de Pesquisa Nuevas Tecnologías en Información de la Universidade Estadual Paulista Júlio de Mesquita Filho (Brasil), Grupo de Investigación en Inteligencia Computacional de la Universidad del Cauca (Colombia), al Conselho Nacional de Desenvolvimento Científico e Tecnológico (Brasil), a Coordenação de Aperfeiçoamento de Pessoal de Nível Superior (Brasil) y a Asociación Universitaria Iberoamericana de Postgrado (España).

S. M. Roa-Martínez participo en la concepción y diseño, análisis e interpretación de los datos, y revisión. S. A. B. G. Vidotti participo de la concepción, revisión y aprobación de la versión final del artículo.

\section{Referencias}

Albert, W. Do web users actually look at ads? A case study of banner ads and eye-tracking technology. In: Annual Conference of the Usability Professionals' Association, 11th., 2002, Orlando. Proceedings [...]. Bloomingdale, IL.: UXPA, 2002.
Baeza-Yates, R.; Rivera Loaiza, C.; Velasco Martín, J. Arquitectura de la información y usabilidad en la web. El Profesional de la Información, v. 13, n. 3, p. 168-178, 2004. Doi: http://dx.doi.org $/ 10.1080 / 13866710412331291886$ 
Bojko, A. Eye tracking the user experience. New York: Rosenfeld Media, 2013.

Broder, A. A taxonomy of web search. ACM Sigir Forum, v. 36, n. 2, p. 3-10, 2002. Doi: http://dx.doi.org/10.1145/792550.792552

Byrne, M. D. et al. Eye tracking the visual search of click-down menus. In: Sigchi Conference on Human Factors in Computing Systems, 1999, Pittsburgh. Proceedings online [...]. New York: ACM, 1999. Doi: http://dx.doi.org/10.1145/302979.303118

Cooke, L. Improving usability through eye tracking research. In: Professional Communication Conference, 2004, Minneapolis. Proceedings online [...]. Minneapolis: IEEE, 2004. Doi: http:// dx.doi.org/10.1109/ipcc.2004.1375297

Cowen, I.; Ball, I. J.; Delin, J. An eye movement analysis of web page usability. In: Human Computer Interaction, 2002. Proceedings online [...]. London: Springer, 2002. p. 317-335. Doi: http://dx.doi.org/10.1007/978-1-4471-0105-5_19

Dias, C. Usabilidade na web: criando portais mais acessíveis. Rio de Janeiro: Alta Books, 2003.

Duchowski, A. Eye tracking methodology: theory and practice. Berlin: Springer Science \& Business Media, 2007. v. 373.

Duchowski, A. T. A breadth-first survey of eye-tracking applications. Behavior Research Methods, Instruments, \& Computers, v. 34, n. 4, p. 455-470, 2002. Doi: http://dx.doi.org/10.3758/ bf03195475

Fitts, P. M.; Jones, R. E.; Milton, J. L. Eye movements of aircraft pilots during instrument-landing approaches. Aeronautical Engineering Review, v. 9, n. 2, p. 24-29, 1950.

Giannotto, E. C. Uso de rastreamento do olhar na avaliação da experiência do tele-usuário de aplicações de TV interativa. Dissertação (Mestrado em Engenharia Elétrica) - Universidade de São Paulo, São Paulo, 2009.

Goldberg, J. H.; Kotval, X. P. Computer interface evaluation using eye movements: methods and constructs. International Journal of Industrial Ergonomics, v. 24, n. 6, p. 631-645, 1999. Doi: http://dx.doi.org/10.1016/s0169-8141(98)00068-7

Goldberg, J. H. et al. Eye tracking in web search tasks: design implications. In: Symposium on Eye Tracking Research \& Applications, 2nd., 2002, New Orleans. Proceedings online [...]. New York: ACM, 2002. Doi: http://dx.doi.org/10.1145/50 7072.507082

Goldberg, J. H.; Wichansky, A. M. Eye tracking in usability evaluation: a practitioner's guide. In: Hyönä, J.; Deubel, H.; Radach, R. (ed.). The Mind's Eye: cognitive and applied aspects of eye movement research. North Holland: Elsevier, 2003. p. 493-516. Doi: http://dx.doi.org/10.1016/b978-044451020-4/5 0027-x

International Organization for Standardization. 9241-210: ergonomics of human system-interaction Part 210: humancentred design for interactive systems. Switzerland: ISO, 2010.

Ivory, M. Y.; Hearst, M. A. The state of the art in automating usability evaluation of user interfaces. ACM Computing Surveys, v. 33, n. 4, p. 470-516, 2001. Doi: http://dx.doi.org/10.11 $45 / 503112.503114$

Jacob, R. J.; Karn, K. S. Eye tracking in human-computer interaction and usability research: ready to deliver the promises. Mind, v. 2, n. 3, p. 4, 2003. Doi: http://dx.doi.org/10. 1016/b978-044451020-4/50031-1

Just, M. A.; Carpenter, P. A. Eye fixations and cognitive processes. Cognitive Psychology, v. 8, n. 4, p. 441-480, 1976. Doi: http://dx.doi.org/10.1016/0010-0285(76)90015-3

Kurzhals, K. et al. Eye tracking evaluation of visual analytics. Information Visualization, v. 15, n. 4, p. 340-358, 2015. Doi: http:// dx.doi.org/10. 1177/1473871615609787

Lund, $H$. Eye tracking in library and information science: a literature review. Library Hi Tech, v. 34, n. 4, p. 585-614, 2016. Doi: http://dx.doi.org/10.1108/lht-07-2016-0085

Lupu, R. G.; Ungureanu, F. A survey of eye tracking methods and applications. Buletinul Institutului Politehnic din lasi, Automatic Control and Computer Science Section, v. 3, p. 71-86, 2013.

Marshall, S. P. Method and apparatus for eye tracking and monitoring pupil dilation to evaluate cognitive activity. Current Assignee: Eyetracking LLC., San Diego State University Research Foundation. US6090051A. Publication date: Mar. 3 1999. Priority date: Jul. 18 2000. Available from: https:// patents.google.com/patent/US6090051A/en. Cited: Dec. 10 2018.

Mello-Thoms, C.; Nodine, C. F.; Kundel, H. L. What attracts the eye to the location of missed and reported breast cancers? In: Symposium on Eye Tracking Research \& Applications, 2nd., 2002, New Orleans. Proceedings online [...]. New Orleans: ACM, 2002. Doi: http://dx.doi.org/10.1145/507093.507095

Nielsen, J.; Loranger, H. Usabilidade na web: projetando websites com qualidade. Rio de Janeiro: Campus, 2007.

Poole, A.; Ball, L. J. Eye tracking in human-computer interaction and usability research: current status and future prospects. In: Ghaoui, C. (ed.). Encyclopedia of Human-Computer Interaction. Pennsylvania: Idea Group Inc., 2005.

Poole, A.; Ball, L. J.; Phillips, P. In search of salience: a responsetime and eye-movement analysis of bookmark recognition. In: People and Computers Design for Life, 18th., 2005, London. Proceedings online [...]. Berlin: Springer, 2005. p. 363-378. Doi: http://dx.doi.org/10.1007/1-84628-062-1_23

Prisacari, A.; Holme, T. Using eye-tracking to test and improve website design. In: International Conference of Design, User Experience, and Usability 2nd., 2013, Las Vegas. Proceedings online [...]. Berlin: Springer, 2013. Doi: http://dx.doi. org/10.1007/978-3-642-39229-0_42

Renshaw, J. A. et al. Designing for visual influence: an eye tracking study of the usability of graphical management information. Human-Computer Interaction, v. 1, p. 144-151, 2003.

Roa-Martínez, S. M. Da information findability à image findability: aportes da polirrepresentação, recuperação e comportamento de busca. 2019. Tese (Doutorado em Ciência da Informação) - Universidade Estadual Paulista, Marília, 2019.

Sharma, C.; Dubey, S. K. Analysis of eye tracking techniques in usability and $\mathrm{HCl}$ perspective. In: International Conference on Computing for Sustainable Global Development, 8th., 2014, 
New Delhi. Proceedings online [...]. New Delhi: IEEE, 2014. Doi: http://dx.doi.org/10.1109/indiacom.2014.6828034

Sibert, L. E.; Jacob, R. J. Evaluation of eye gaze interaction. In: Sigchi Conference on Human Factors in Computing Systems, 2000, The Hague. Proceedings online [...]. New York: ACM, 2000. Doi: http://dx.doi.org/10.1145/332040

Universidade Estadual Paulista. Repositório Institucional Unesp. São Paulo, 2019. Disponível em: https://repositorio.unesp.br/. Acesso em: 10 dez. 2018.

Vermeeren, A. P. O. S. et al. User experience evaluation methods: current state and development needs. In: Nordic Conference on Human-Computer Interaction: Extending
Boundaries Systems, 6th., 2010, Reykjavik Iceland. Proceedings online [...]. New York: ACM, 2010. p. 521-530. Doi: http://dx.doi. org/10.11 45/1868914.1868973

Vidotti, S. A. B. G. et al. Arquitetura da informação e eye tracking: o que o olhar e os dados revelam. Informação \& Tecnologia, v. 3, n. 1, p. 181-202, 2016. Doi: http://dx.doi.org/10.22478/ ufpb.1981-0695.2018v13n2.42941

Vuori, T. et al. Can eye movements be quantitatively applied to image quality studies? In: Nordic Conference on HumanComputer Interaction, 3th., 2004, Tampere. Proceedings online [...]. New York: ACM, 2004. Doi: http://dx.doi.org/10. 1145/1028014.1028067 